UN MODELO DE COINTEGRACIÓN PARA PRONOSTICAR EL PIB DE MÉXICO

Alejandro Pérez-López Elguezabal ${ }^{1}$

DOCUMENTO DE INVESTIGACIÓN NO. 9504

JULIO, 1995

Banco de México. Las opiniones expresadas por el autor pueden no corresponder a las de la institución. El autor agradece los comentarios del grupo de trabajo de la Dirección de Estudios Económicos y a Maricarmen Hernández por su valiosa cooperación en la elaboración de este trabajo. 


\title{
UN MODELO DE COINTEGRACIÓN PARA PRONOSTICAR EL PIB DE MÉXICO
}

\author{
ALEJANDRO PÉREZ-LÓPEZ ELGUEZABAL \\ JULIO 1995
}

DOCUMENTO DE INVESTIGACIÓN NO. 9504

\section{RESUMEN}

El propósito de este trabajo es construir un modelo econométrico para pronosticar el producto interno bruto. Se presenta un modelo de equilibrio general de una economía abierta de dos sectores. A partir de este modelo se deriva una relación inversa entre producción y tipo de cambio real. Tanto el índice de volumen de la producción industrial como el tipo de cambio real bilateral México-Estados Unidos resultan ser variables no-estacionarias. No obstante ambas variables están cointegradas. La regresión de tipo de cambio real bilateral y se pronosticó la producción industrial para los tres últimos trimestres de 1995. Luego a partir de estos pronósticos, y a través de una regresión auxiliar, se pronosticó el producto interno bruto de 1995. 
UN MODELO DE COINTEGRACIÓN PARA PRONOSTICAR EL PIB DE MÉXICO

ALEJANDRO PÉREZ-LÓPEZ ELGUEZABAL

JULIO 1995

DOCUMENTO DE INVESTIGACIÓN NO. 71

\section{Contenido}

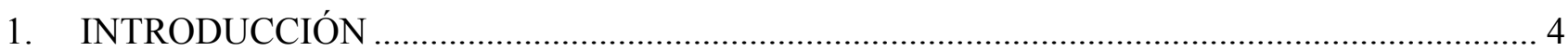

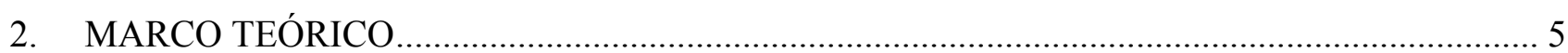

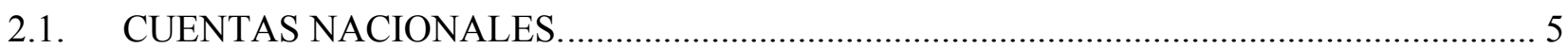

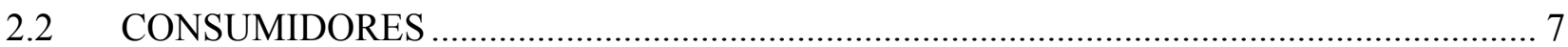

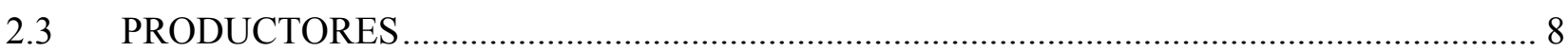

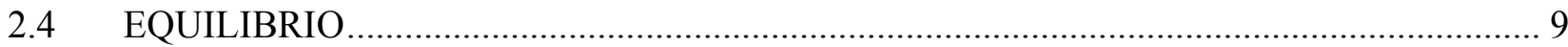

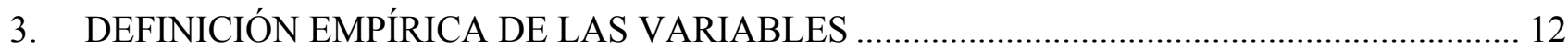

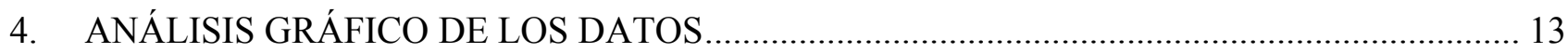

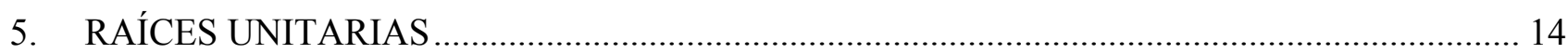

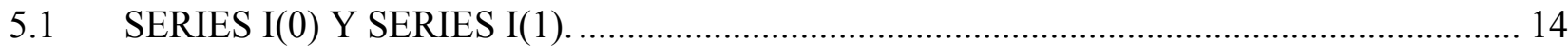

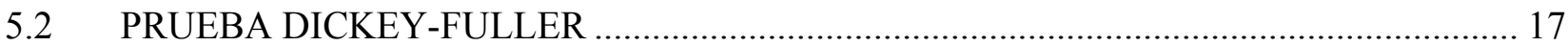

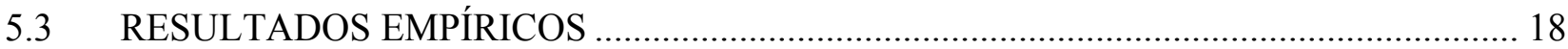

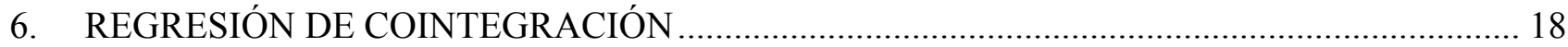

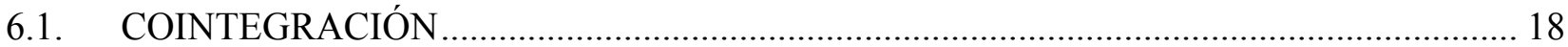

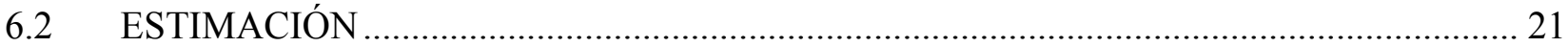

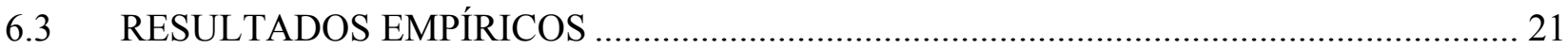

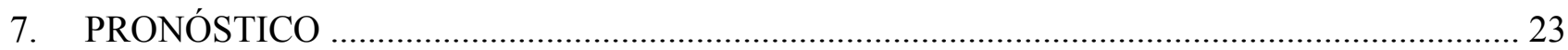

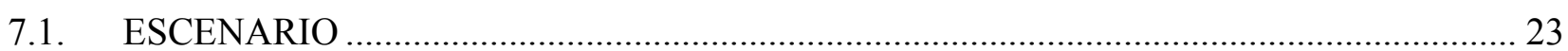

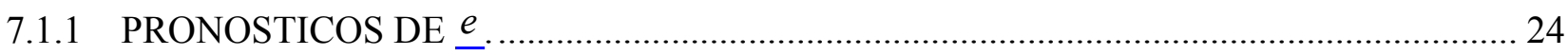

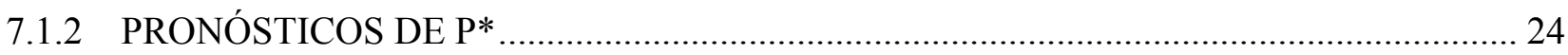

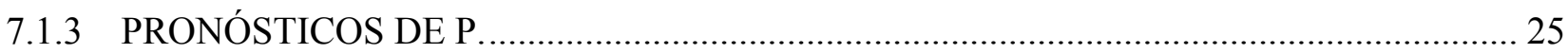

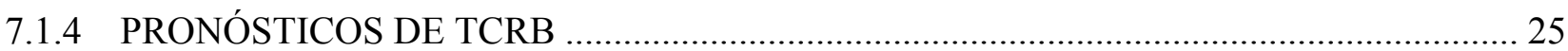

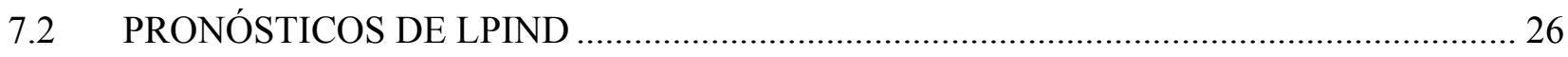

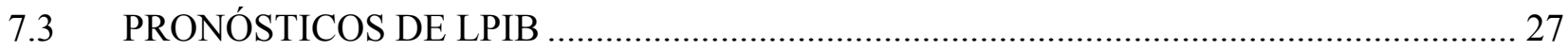

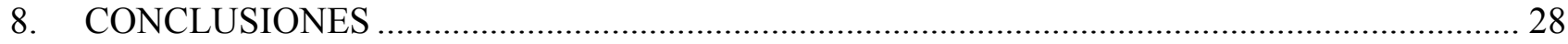

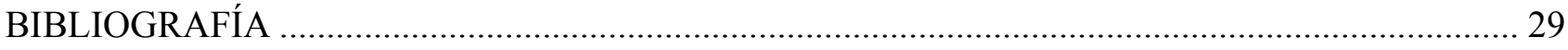




\section{UN MODELO DE COINTEGRACIÓN PARA PRONOSTICAR EL PIB DE MÉXICO}

\section{INTRODUCCIÓN}

El propósito de este trabajo es construir un modelo econométrico para pronosticar el producto interno bruto trimestral.

En la sección 2 se presenta un modelo de equilibrio general de una economía abierta de dos sectores: un sector es semi-comerciable y el otro es comerciable. La oferta agregada del bien semi-comerciable como función del precio relativo, sirve de base para establecer una relación funcional entre producto interno bruto y el tipo de cambio real multilateral.

En la sección 3 se definen empíricamente las variables, la muestra de datos abarca del primer trimestre de 1980 al primer trimestre de 1995. Es conveniente sustituir las variables producto interno bruto e índice del tipo de cambio real multilateral por las variables instrumentales índice de volumen de la producción industrial e índice del tipo de cambio real bilateral México-Estados Unidos respectivamente. En la sección 4 se analizan gráficamente los datos. Las gráficas indican una aparente relación negativa y estable entre la producción industrial y el tipo de cambio real bilateral.

En la sección 5 se presentan pruebas de raíz unitaria de la producción industrial y del tipo de cambio real bilateral, para establecer si las series son estacionarias o no. En la sección 6 se corre la regresión de cointegración entre la producción industrial y el tipo de cambio real bilateral, y se hace la prueba de cointegración.

En la sección 7 se utiliza la regresión de cointegración para calcular los pronósticos. Se forma un escenario futuro para el tipo de cambio real bilateral y se pronostica la producción industrial para los tres últimos trimestres de 1995. Luego a partir de estos pronósticos, y a través de una regresión auxiliar, se pronostica el producto interno bruto para 1995. Por último, las conclusiones se presentan en la sección 8 . 


\section{MARCO TEÓRICO}

El propósito de esta sección es construir un modelo sencillo de equilibrio general que sirva de base para la estimación econométrica de la oferta agregada. [Mussa (1986)].

\subsection{CUENTAS NACIONALES.}

Hay dos bienes en la economía: X e y. El bien x es semi-comerciable, se produce domésticamente, se consume domésticamente y se exporta. El bien y es comerciable y únicamente se importa debido a que no se produce domésticamente.

La restricción presupuestal de los consumidores, gastos igual a ingresos, es

$$
p x^{d}+y^{d}=\pi+w l^{s}-t
$$

donde $x^{d} e y^{d}$ son las cantidades demandadas de los bines, $\mathrm{p}$ es el precio relativo de $\mathrm{x}$ en términos de $\mathrm{y}$, $\pi$ son los beneficios de las empresas, $\mathrm{x}$ es el salario, $\ell^{s}$ es la cantidad ofrecida de trabajo y $\mathrm{t}$ son los impuestos.

El estado de resultados de las empresas, beneficios igual a ingresos menos costos es

$$
\pi=p x^{s}-w \ell^{d}
$$

donde $\chi^{\mathrm{s}}$ es la cantidad ofrecida del bien semi-comerciable y $\ell^{d}$ es la cantidad demandada de trabajo.

La restricción presupuestal del gobierno, gasto igual a recaudación, es:

$$
p x^{g}+y^{g}=t
$$

donde $\chi^{\mathrm{g}}$ e $\mathrm{y}^{\mathrm{g}}$ son los gastos de gobierno en ambos bienes: 
Se pueden consolidar los tres estados financieros, al sustituir $\pi$ de (2) y $t$ de (3) en (1) se obtiene la Ley de Walras.

$$
p\left[x^{s}-x^{d}-x^{g}\right]-y^{d}-y^{g}+w\left[\ell^{s}-\ell^{d}\right]=0
$$

la suma de excesos de demanda es cero.

Si el mercado de trabajo está en equilibrio $\ell^{s}=\ell^{d}$ entonces necesariamente el valor de las exportaciones es igual a las importaciones, $p\left[x^{s}-x^{d}-x^{g}\right]=y^{d}+y^{g}$

Sin embargo, para determinar el valor de equilibrio del precio relativo p es necesario especificar la demanda externa del bien $\mathrm{x}$, la cual se supone que es

$$
\chi^{* \mathrm{~d}}(\mathrm{p}, \mathrm{gnp})
$$

$$
(-)(+)
$$

la demanda externa es una función decreciente en el precio relativo p y creciente en el "gross national product".

El equilibrio de esta economía se logra cuando simultáneamente el mercado de trabajo y el mercado del bien semi-comerciable están en equilibrio, es decir

$$
\begin{gathered}
\ell^{d}=\ell^{s} \\
\chi^{* d}=\chi^{\mathrm{s}}-\chi^{\mathrm{d}}-\chi^{\mathrm{g}}
\end{gathered}
$$




\section{Gráfica 1}

Equilibrio en el Mercado de Trabajo

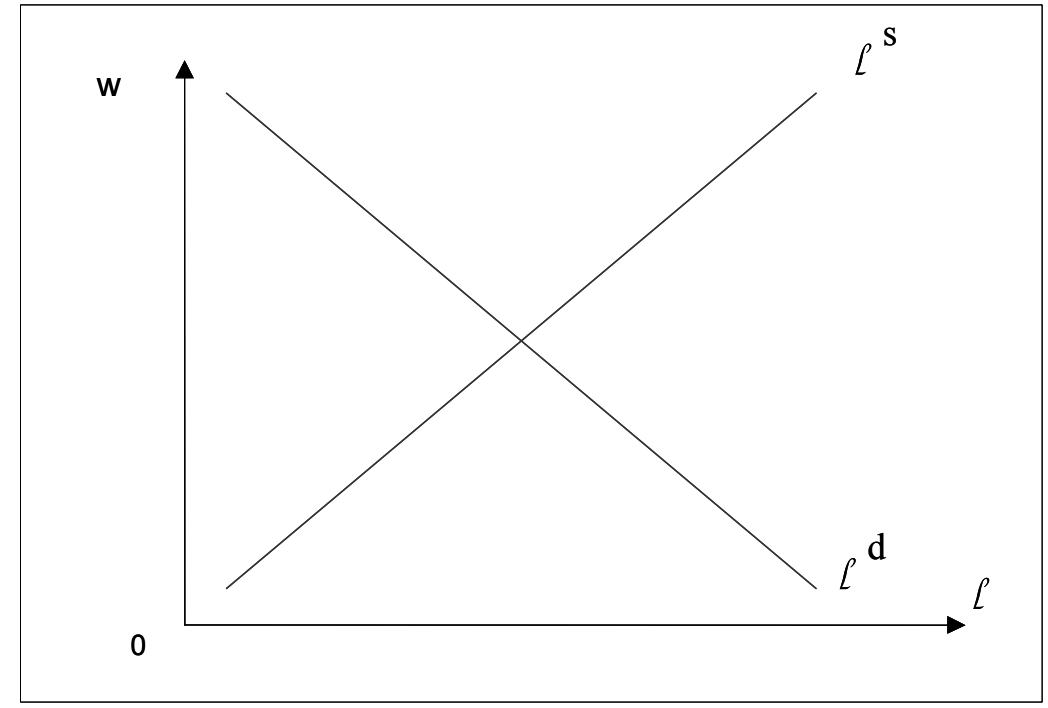

Gráfica

Equilibrio en el Mercado del Bien Semi-Comerciable

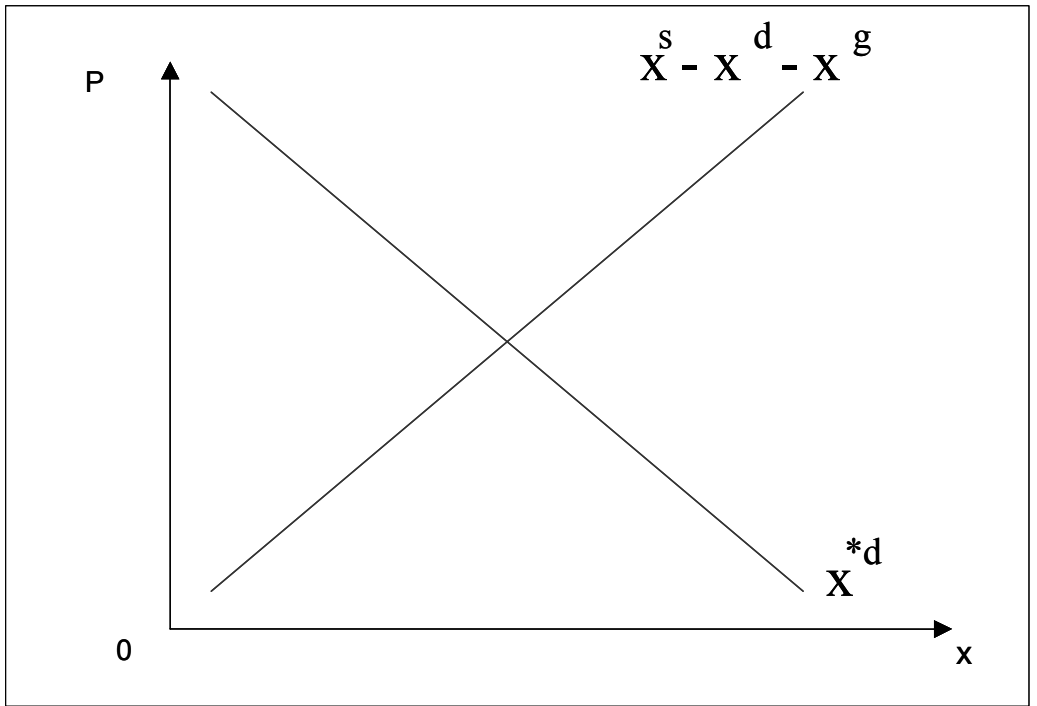

\subsection{CONSUMIDORES}

Las demandas privadas de bienes así como la oferta de trabajo se derivan de la maximización de utilidad sujeta a la restricción presupuestal por parte de los consumidores.

Más específicamente el problema de los consumidores es el siguiente 
$\operatorname{Max} U(x, y, \bar{\ell}-\ell)$

s.a. $\quad p x+y=\pi+w \ell-t$

donde $\bar{\ell}$ es la dotación de trabajo.

Como resultado de la maximización se obtienen las demandas de bienes

$$
\begin{array}{rr}
x^{d}(p, \pi, w) & \mathrm{y}^{\mathrm{d}}(p, \pi, w) \\
(-)(+)(+) & (+)(+)(+)
\end{array}
$$

y la oferta de trabajo

$$
\begin{gathered}
\ell^{s}(p, \pi, w) \\
(-)(-)(+)
\end{gathered}
$$

La estática comparativa está dada por los signos debajo de las demandas y de la oferta. Por ejemplo $\chi^{\mathrm{d}}$ es decreciente en su propio precio y $\ell^{s}$ es creciente en el salario. En la estática comparativa se supone que los efectos sustitución dominan a los efectos ingreso.

\subsection{PRODUCTORES}

Dadas las condiciones de producción representadas en la función producción, los productores maximizan beneficios.

La función producción que relaciona la cantidad producida con la cantidad de trabajo es:

$$
x=f(\ell)
$$

la cual se supone creciente $f^{\prime}>0$ y de rendimientos decrecientes $f^{\prime \prime}<0$

Por consiguiente, el problema de los productores es el siguiente

$$
\max \{p f(\ell)-w \ell\}
$$


De la maximización de beneficios se obtienen la demanda de trabajo

$$
\begin{aligned}
& \chi^{\mathrm{s}}(\mathrm{p}, \mathrm{w}) \\
& (+)(-)
\end{aligned}
$$

Al evaluar la función objetivo en la cantidad óptima de trabajo se obtiene la función de beneficios

$$
\pi(\mathrm{p}, \mathrm{w})
$$

$(+)(-)$

\subsection{EQUILIBRIO}

La condición de equilibrio en el mercado de trabajo es:

$$
\ell^{d}(p, w)=\ell^{s}[p, \pi(p, w), w]
$$

\section{Gráfica 3}

Equilibrio en el Mercado de Trabajo

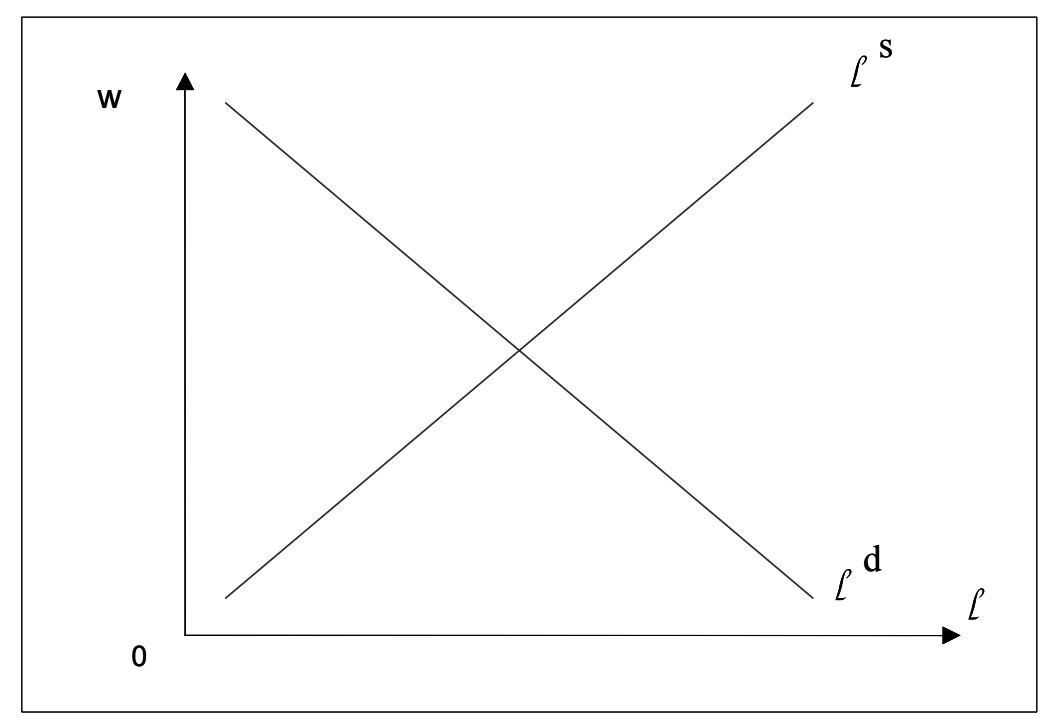


El salario de equilibrio depende del precio relativo, lo cual genera una función implícita entre w y $p$.

$\mathrm{w}(\mathrm{p})$

Por otra parte, la condición de equilibrio en el mercado del bien semi-comerciable es

$$
\begin{aligned}
x^{d}[p, \pi(p, w), w] & +x^{* d}(p, g n p)+x^{g} \\
& =x^{s}[p, \pi(p, w)]
\end{aligned}
$$

Utilizando la ecuación (15) la condición anterior se puede reexpresar como

$$
x^{d}(p)+x^{* d}(p, g n p)+x^{g}=x^{s}(p)
$$

donde gnp y $\chi^{\mathrm{g}}$ son variables Exógenas, y p es la única variable endógena.

Si se considera al sistema de ecuaciones simultáneas anterior desde el punto de vista econométrico, se tiene que la ecuación de oferta está identificada mientras que la ecuación de demanda está subidentificada. Es decir, las observaciones se tienden a distribuir a lo largo de la ecuación de oferta agregada.

\section{Gráfica 4}

Equilibrio en el Mercado General

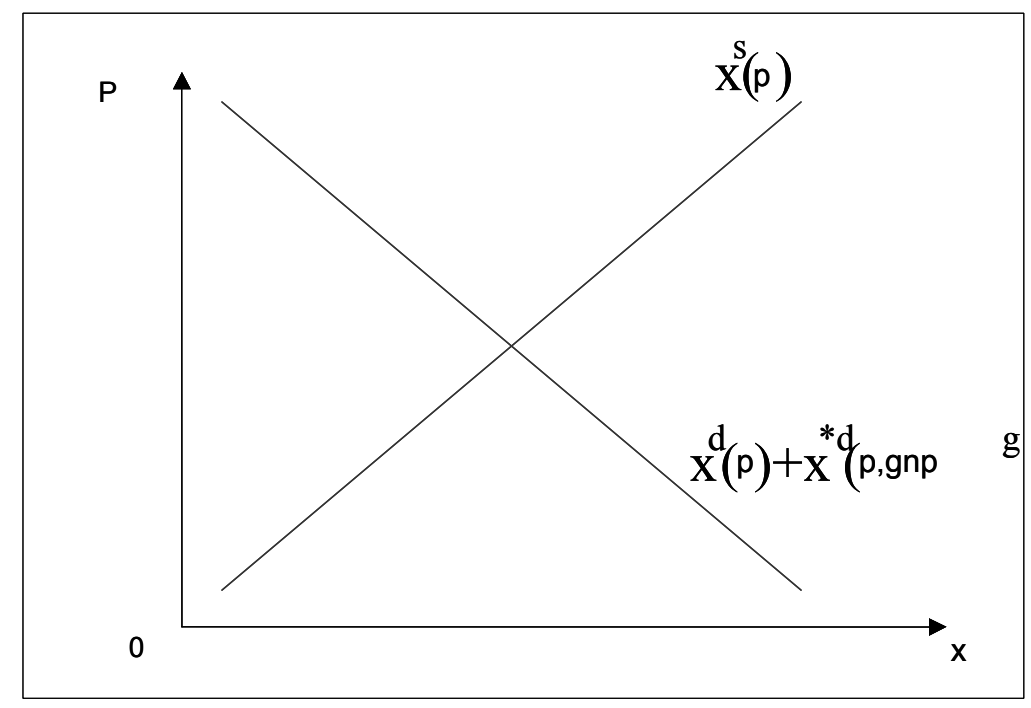


Por otra parte, el precio relativo $p$ es el inverso del tipo de cambio real.

\section{$p=$ precio (interno) del bien semi-comerciable}

[Tipo de cambio] [precio del bien comerciable]

es decir

$$
p=\frac{1}{T C R}
$$

por lo consiguiente, la oferta agregada es decreciente en el tipo de cambio real.

\section{Gráfica 5}

Oferta Agregada en Función del Tipo de Cambio Real

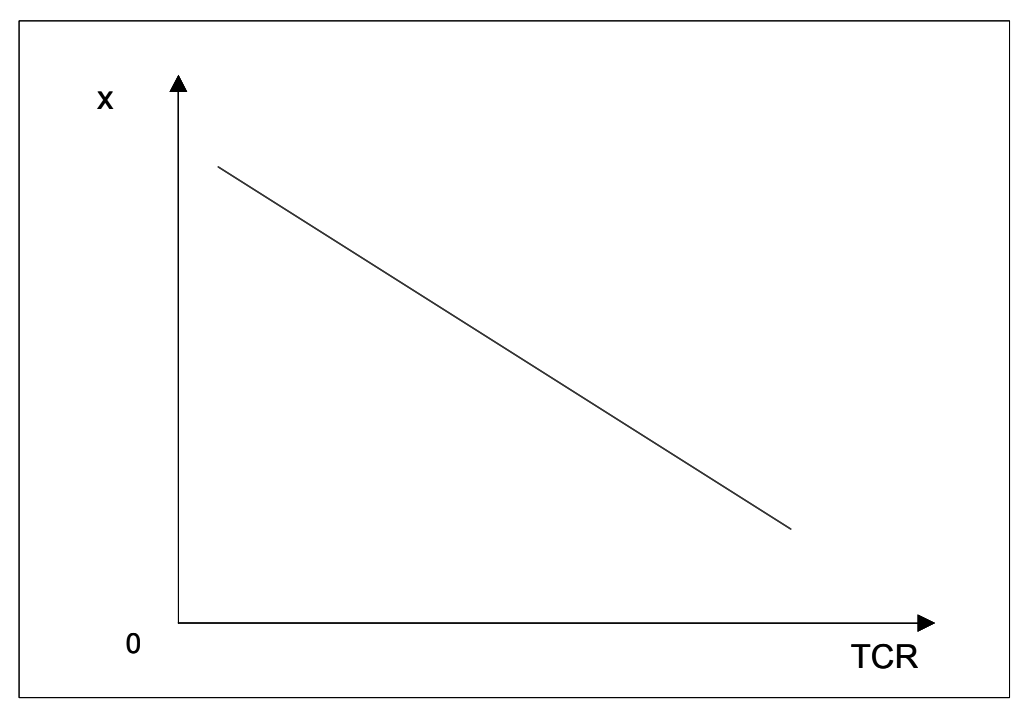




\section{DEFINICIÓN EMPÍRICA DE LAS VARIABLES}

Los datos son trimestrales, la muestra abarca del primer trimestre de 1980 al primer trimestre de 1995.

Los datos de producto son

LPIB $=$ Logaritmo del producto interno bruto trimestral.
LPING $=$ Logaritmo del índice de volumen de la producción industrial.

En la gráfica 6 se presentan las variables LPIB y LPIND. La correlación entre las dos variables es .9563 .

Los datos de tipo de cambio real son

LTCRM = Logaritmo del tipo de cambio real multilateral de México y 133 países.

LTCTM = Logaritmo del tipo de cambio real de México y Estados Unidos.

En la gráfica 7 se muestran las variables LTCRM y LTCRB. La correlación entre las dos series es .8755 .

En principio las variables x y $1 / \mathrm{p}$ del merco teórico corresponden a las series LPIB y LTCRM. Sin embargo, es preferible reemplazar las variables LPIB y LTCRM por las variables LPIND y LTCRB, debido a que para estas últimas los datos se obtienen con menor retraso, y debido a que la serie LPBI tiene bastante estacionalidad lo cual dificulta la estimación. 
Gráfica 6

LPIB y LPIND

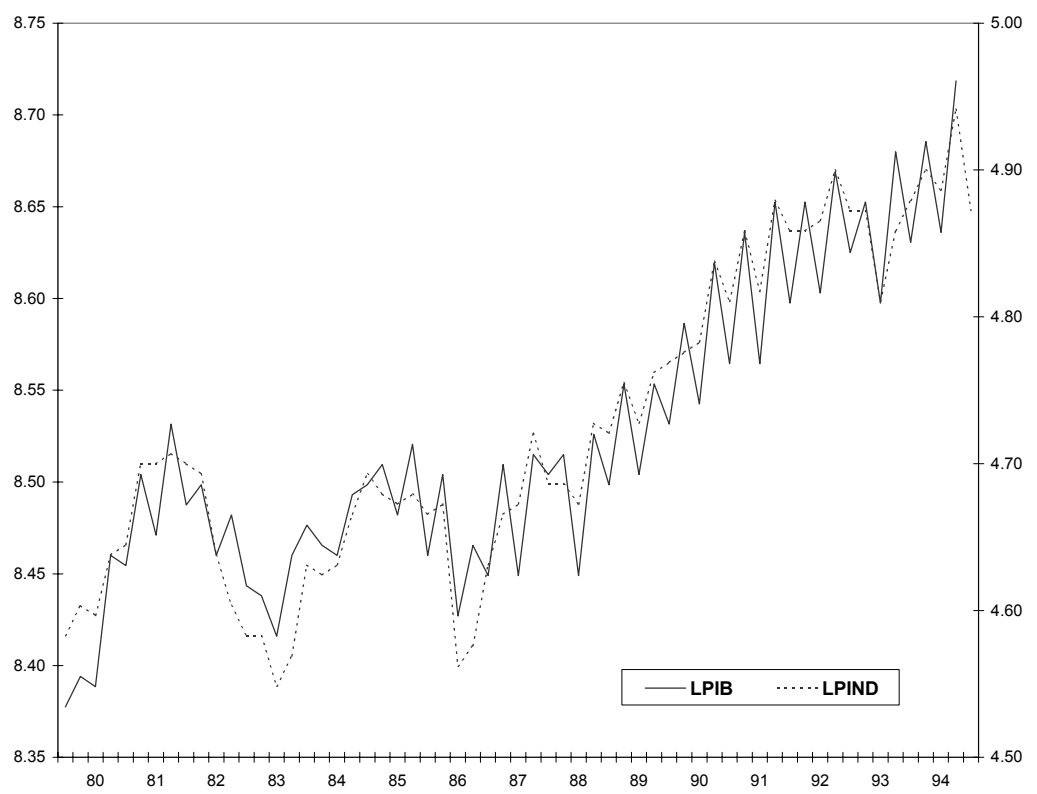

Gráfica 7

LTCRM y LTCRB

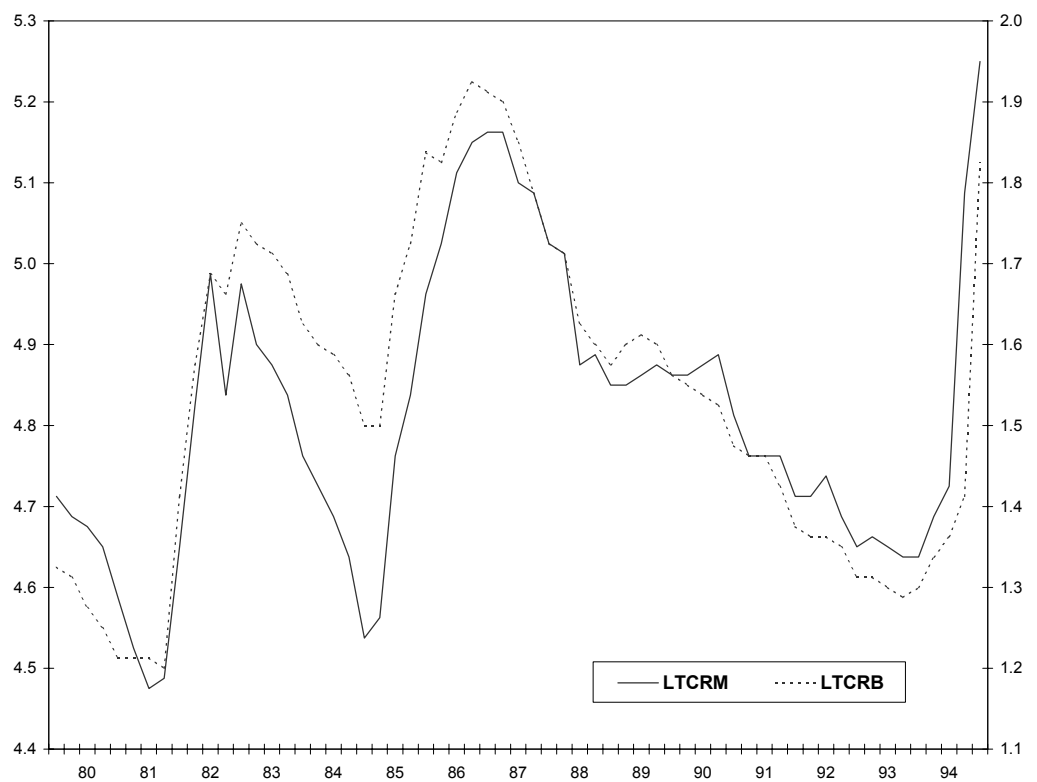

\section{ANÁLISIS GRÁFICO DE LOS DATOS}

Antes de efectuar las estimaciones econométricas es conveniente hacer un análisis gráfico de los datos. 
En la gráfica 8 se grafican el logaritmo del índice de volumen de la producción industrial sin tendencia LPINDR y el logaritmo del índice del tipo de cambio real bilateral LTCRB contra el tiempo.

En la gráfica 9 se grafica el logaritmo del índice de volumen de la producción industrial sin tendencia LPINDR contra el logaritmo del índice de tipo de cambio real bilateral LTCRB.

Se puede aprecia en ambas gráficas que existe una relación inversa entre las dos series, al revaluarse (disminuir) el tipo de cambio real bilateral aumenta el índice de volumen de la producción industrial. La correlación entre las dos series es -.7914.

\section{RAÍCES UNITARIAS}

Al correr una regresión es importante saber si la variable dependiente y la variable independiente son series estacionarias o no. Las propiedades de los estimadores son diferentes cuando las series son estacionarias que cuando no lo son. Por lo consiguiente antes de correr la regresión entre el producto y el tipo de cambio real, es necesario investigar individualmente cada serie de tiempo.

A continuación se presenta una manera formal de clasificar las series de tiempo en series estacionarias y no estacionarias, así como una prueba estadística para establecer si determinada serie de tiempo es estacionaria o no, y por último se presentan los resultados de aplicar la prueba a las series de producción industrial y de tipo de cambio real. [Engle y Granger (1991)].

\subsection{SERIES I(0) Y SERIES I(1).}

Para simplificar se consideran dos tipos de series de tiempo, series $\mathrm{I}(0)$ que son estacionarias y series I(1) que no lo son.

En una serie $\mathrm{I}(0)$ un shock pasado no tiene prácticamente ningún efecto en el valor corriente de la serie, si el shock es suficientemente lejano. En una serie I(1) cualquier shock pasado tiene un efecto sobre el valor corriente de la serie.

Se supone que la serie $\chi_{t}$ se genera de la manera siguiente 


$$
x_{t}=\sum_{j=0}^{\infty} \alpha_{j} \varepsilon_{t-j}
$$

\section{Gráfica 8}

LPINDR y LTCRB

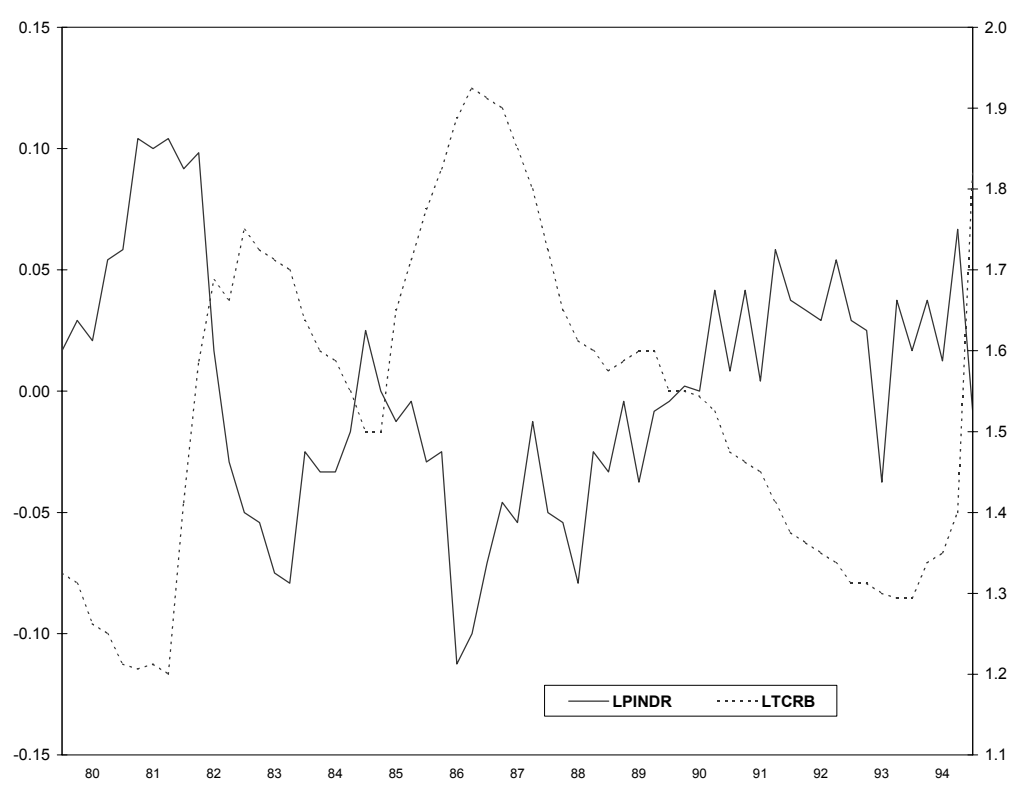

Gráfica 9

LPINDRY y LTCRB

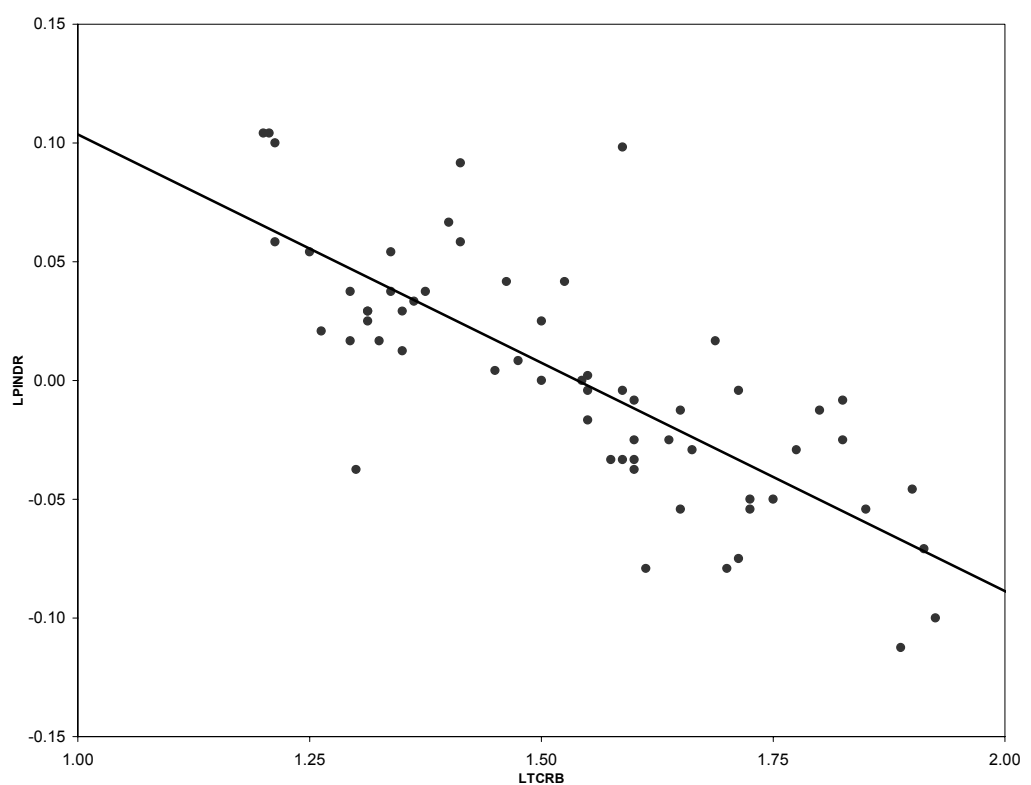


Donde $\varepsilon_{\mathrm{t}}$ es una serie de innovaciones independientes e idénticamente distribuidas. Si la serie es I(9) entonces $\alpha_{j}$ tiende a cero cuando j aumenta. Si la serie es $I(1)$ entonces $\alpha_{j}$ no tiende a cero cuando j aumenta.

Como ejemplo consideren proceso autoregresivo de primer orden.

$$
\chi_{\mathrm{t}}=\alpha \chi_{\mathrm{t}-1}+\varepsilon_{\mathrm{t}}
$$

En un primer caso, cuando $-1<\mathrm{a}<1$, se tiene que

$$
x_{t} \sum_{j=0}^{\infty} \alpha^{j} \varepsilon_{t-j}
$$

de manera que el coeficiente de $\varepsilon_{t-j}$ tiende a cero exponencialmente, por lo tanto la serie es $\mathrm{I}(0)$.

En un segundo caso, cuando se supone a=1, la serie es un "random walk" que se puede expresar como

$$
x_{t} \sum_{j=0}^{\infty} \alpha^{j} \varepsilon_{t-j}
$$

donde los shocks antiguos tienen la misma ponderación que los shocks nuevos en la determinación del valor corriente de $\chi$, por lo consiguiente la serie es $\mathrm{I}(1)$.

En este último caso se tiene que

$$
\Delta \chi_{\mathrm{t}}=\varepsilon_{\mathrm{t}}
$$

es decir, el incremento de una variable I(1) es una variable I(0). 
Una serie $\mathrm{I}(0)$ tiene media y varianza constante, de manera que tiene una tendencia a regresar y cruzar frecuentemente su valor medio. Una serie I(1) no tiende a regresar a ningún valor, y su varianza aumenta linealmente con el tiempo.

\subsection{PRUEBA DICKEY-FULLER}

La prueba estadística Dickey-Fuller sirve para establecer si determinada serie de tiempo es noestacionaria.

Para probar si una serie es I(1) se considera el siguiente modelo autoregresivo

$$
y_{t}=p y_{t-1}+\varepsilon_{t}
$$

que implica

$$
\Delta y_{t}=(p-1) y_{t-1}+\varepsilon_{t}
$$

La hipótesis nula es

La hipótesis alternativa es

$$
\begin{aligned}
& H_{0}:(p-1)=0 \\
& H_{A}:(p-1)<0
\end{aligned}
$$

El valor del coeficiente t de la regresión (17) es el estadístico Dickey-Fuller.

En la práctica se puede incluir una constante en la regresión (17), pero esto altera la distribución de la prueba estadística, y lo mismo ocurre si se incluye una tendencia lineal.

Una segunda cuestión práctica es que para la mayoría de las series $\varepsilon_{\mathrm{t}}$ no es ruido blanco. La solución sugerida por Dickey y Fuller es aumentar la ecuación de regresión (17) añadiendo suficientes términos $\Delta \mathrm{y}_{\mathrm{t}-1}$ para "blanquear" los residuales.

La prueba de Dickey-Fuller aumentada es el coeficiente $t$ de la variable $\mathrm{y}_{\mathrm{t}-1}$ en:

$$
\Delta y_{t}=\delta_{0}+\delta_{t} t+(p-1) y_{t-1}+\sum \Theta_{i} \Delta y_{t-1}+\varepsilon_{t}
$$




\subsection{RESULTADOS EMPÍRICOS}

El cuadro 1 presenta los resultados de la prueba Dickey-Fuller para la serie del logaritmo de la producción industrial LPIND. De manera similar el cuadro 2 presenta los resultados para la serie del logaritmo del tipo de cambio real LTCRB.

Se presentan diferentes pruebas, sin constante, con constante y con tendencia lineal. Asimismo, se presentan pruebas sin rezagos, y con un rezago.

En todas las pruebas no se rechaza la hipótesis nula. Por lo consiguiente las pruebas indican que tanto el logaritmo de la producción industrial LPIND como el logaritmo del tipo de cambio real LTCRB son series I(1), es decir, son series no-estacionarias.

\section{REGRESIÓN DE COINTEGRACIÓN}

En esta sección se presenta el concepto de cointegración entre dos series de tiempo por medio de la regresión de cointegración. También se discute brevemente la forma de estimar una regresión de cointegración. Por último, se presentan las estimaciones empíricas de la regresión de cointegración entre el logaritmo de la producción industrial LPIND y el logaritmo del tipo de cambio real LTCRB.

\subsection{COINTEGRACIÓN}

Según la teoría económica, ciertas variables no deben alejarse mucho unas de otras en el largo plazo. Tales variables pueden alejarse en el corto plazo pero existen fuerzas económicas, ya sea mecanismos de mercado o intervenciones del gobierno, que tienden a juntarlas en el largo plazo. Por ejemplo: las tasas de interés, los precios de un mismo bien en diferentes localidades de un país, ingresos y gastos del gobierno, oferta de dinero y nivel de precios, etc.

Si se tiene un par de series $\chi_{t}$ e $y_{t}$ cada una de las cuales es I(1), en general una combinación lineal de estas series es también $\mathrm{I}(1)$. Sin embargo, si existe una constante A tal que

$$
z_{t}=y_{t}-A x_{t}
$$


sea I (0), entonces $x_{t}$ e $y_{t}$ deben tener una relación muy especial. La relación

\section{Cuadro 1}

\section{Prueba Dickey-Fuller de LPIND}

\begin{tabular}{|c|c|c|}
\hline \multicolumn{3}{|l|}{ Sin constante, sin rezagos:LPIND } \\
\hline \multicolumn{3}{|l|}{ Estadístico Dickey-Fuller } \\
\hline \multirow[t]{4}{*}{ Valores Críticos (MacKinnon) } & $1 \%$ & 1.0437 \\
\hline & $5 \%$ & -2.6019 \\
\hline & $10 \%$ & -1.9460 \\
\hline & & -1.6187 \\
\hline \multicolumn{3}{|l|}{ Sin constante, un rezago: LPIND } \\
\hline Estadístico Dickey-Fuller & & 1.5684 \\
\hline \multirow[t]{3}{*}{ Valores Críticos (MacKinnon) } & $1 \%$ & -2.6019 \\
\hline & $5 \%$ & -1.9470 \\
\hline & $10 \%$ & -1.6187 \\
\hline \multicolumn{3}{|l|}{ Constante, sin rezagos: LPIND } \\
\hline Estadístico Dickey-Fuller & & -1.1022 \\
\hline \multirow[t]{3}{*}{ Valores Críticos (MacKinnon) } & $1 \%$ & -3.5437 \\
\hline & $5 \%$ & -2.9109 \\
\hline & $10 \%$ & -2.5928 \\
\hline \multicolumn{3}{|l|}{ Constante, un rezagos: LPIND } \\
\hline Estadístico Dickey-Fuller & & -0.5795 \\
\hline \multirow[t]{3}{*}{ Valores Críticos (MacKinnon) } & $1 \%$ & -3.5437 \\
\hline & $5 \%$ & -2.9109 \\
\hline & $10 \%$ & -2.5928 \\
\hline \multicolumn{3}{|l|}{ Tendencia, sin rezagos: LPIND } \\
\hline Estadístico Dickey-Fuller & & -2.5865 \\
\hline \multirow[t]{3}{*}{ Valores Críticos (MacKinnon) } & $1 \%$ & -4.1190 \\
\hline & $5 \%$ & -3.4862 \\
\hline & $10 \%$ & -3.1711 \\
\hline \multicolumn{3}{|l|}{ Tendencia, un rezagos: LPIND } \\
\hline Estadístico Dickey-Fuller & & -1.8852 \\
\hline \multirow[t]{3}{*}{ Valores Críticos (MacKinnon) } & $1 \%$ & -4.1190 \\
\hline & $5 \%$ & -3.4862 \\
\hline & $10 \%$ & -3.1711 \\
\hline
\end{tabular}




\section{Cuadro 2}

Prueba Dickey-Fuller de LTCRB

\begin{tabular}{|c|c|c|}
\hline \multicolumn{3}{|l|}{ Sin constante, sin rezagos:LTCRB } \\
\hline Estadístico Dickey-Fuller & & 0.6615 \\
\hline \multirow[t]{3}{*}{ Valores Críticos (MacKinnon) } & $1 \%$ & -2.6019 \\
\hline & $5 \%$ & -1.9460 \\
\hline & $10 \%$ & -1.6187 \\
\hline \multicolumn{3}{|l|}{ Sin constante, un rezago: LTCRB } \\
\hline Estadístico Dickey-Fuller & & 0.5982 \\
\hline \multirow[t]{3}{*}{ Valores Críticos (MacKinnon) } & $1 \%$ & -2.6019 \\
\hline & $5 \%$ & -1.9460 \\
\hline & $10 \%$ & -1.6187 \\
\hline \multicolumn{3}{|l|}{ Constante, sin rezagos: LTCRB } \\
\hline Estadístico Dickey-Fuller & & -1.3782 \\
\hline \multirow[t]{3}{*}{ Valores Críticos (MacKinnon) } & $1 \%$ & -3.5437 \\
\hline & $5 \%$ & -2.9109 \\
\hline & $10 \%$ & -2.5928 \\
\hline \multicolumn{3}{|l|}{ Constante, un rezagos: LTCRB } \\
\hline Estadístico Dickey-Fuller & & -2.0876 \\
\hline \multirow[t]{3}{*}{ Valores Críticos (MacKinnon) } & $1 \%$ & -3.5437 \\
\hline & $5 \%$ & -2.9109 \\
\hline & $10 \%$ & -2.5928 \\
\hline \multicolumn{3}{|l|}{ Tendencia, sin rezagos: LTCRB } \\
\hline Estadístico Dickey-Fuller & & -1.3611 \\
\hline \multirow[t]{3}{*}{ Valores Críticos (MacKinnon) } & $1 \%$ & -4.1190 \\
\hline & $5 \%$ & -3.4862 \\
\hline & $10 \%$ & -3.1711 \\
\hline \multicolumn{3}{|l|}{ Tendencia, un rezagos: LTCRB } \\
\hline Estadístico Dickey-Fuller & & -1.9706 \\
\hline \multirow[t]{3}{*}{ Valores Críticos (MacKinnon) } & $1 \%$ & -4.1190 \\
\hline & $5 \%$ & -3.4862 \\
\hline & $10 \%$ & -3.1711 \\
\hline
\end{tabular}




$$
\mathrm{Y}_{\mathrm{t}}=\mathrm{A} \chi_{\mathrm{t}}
$$

Se puede considerar una relación de largo plazo o de equilibrio.

\subsection{ESTIMACIÓN}

Para estimar una regresión de cointegración, Engle y Granger sugieren correr una regresión con las dos variables utilizando mínimos cuadrados. La regresión de cointegración es

$$
y_{t}=\hat{\alpha} x_{t}+z_{t}
$$

donde $\mathrm{z}_{\mathrm{t}}$ representa los residuales.

El método de mínimos cuadrados en efecto logra una buena estimación del coeficiente de cointegración. El estimador de mínimos cuadrados es consistente, y no sólo eso sino que se puede demostrar que el estimador converge a su valor verdadero a una tasa $\mathrm{T}^{-1}$ en vez de $\mathrm{T}^{-1 / 2}$ de manera que el estimador de mínimos cuadrados es superconsistente. Si dos series están cointegradas, es fácil encontrar la relación verdadera. Para lograr una estimación consistente no es necesario considerar problemas de simultaneidad o autocorrelación.

Para probar si dos series están cointegradas, primero hay que probar que las series son integradas individualmente, luego correr la regresión de cointegración y aplicar la prueba de Dickey-Fuller a los residuales. Las pruebas estadísticas difieren si la regresión de cointegración incluye una constante y/o una tendencia lineal.

\subsection{RESULTADOS EMPÍRICOS}

La regresión cointegración es: 


\section{Cuadro 3}

\section{Regresión de Cointegración}

\begin{tabular}{|c|c|c|c|}
\hline \multicolumn{4}{|c|}{ VARIABLE DEPENDIENTE LPIND } \\
\hline \multicolumn{4}{|c|}{ MUESTRA: 1980.1 A 1995.1} \\
\hline \multicolumn{4}{|c|}{ NÚMERO DE OBSERVACIONES 61} \\
\hline VARIABLE & COEFICIENTE & ERROR ESTAND & ESTADÍSTICO $\mathrm{t}$ \\
\hline Constante & 4.8733 & 0.0324 & 150.3356 \\
\hline Tendencia & 0.0051 & 0.0002 & 22.1954 \\
\hline LTCRB & -0.1997 & 0.0201 & -9.9319 \\
\hline R cuadrada & \multicolumn{2}{|c|}{0.9152 Media de la var dep } & 4.7256 \\
\hline$R$ cuadrada ajust & \multicolumn{2}{|c|}{0.9123 Desv est de la var dep } & 4.7256 \\
\hline Log Likelihood & \multicolumn{2}{|c|}{ 125.6671 Suma de resid al cuad } & 0.058 \\
\hline Durbin-Watson & \multicolumn{2}{|c|}{0.9936 Estadístico $\mathrm{F}$} & 313.015 \\
\hline
\end{tabular}

La gráfica 10 presenta los valores observados de LPIND, los valores calculados por la regresión y los residuales.

\section{Gráfica 10}

Valores Observados, Calculados y Residuales

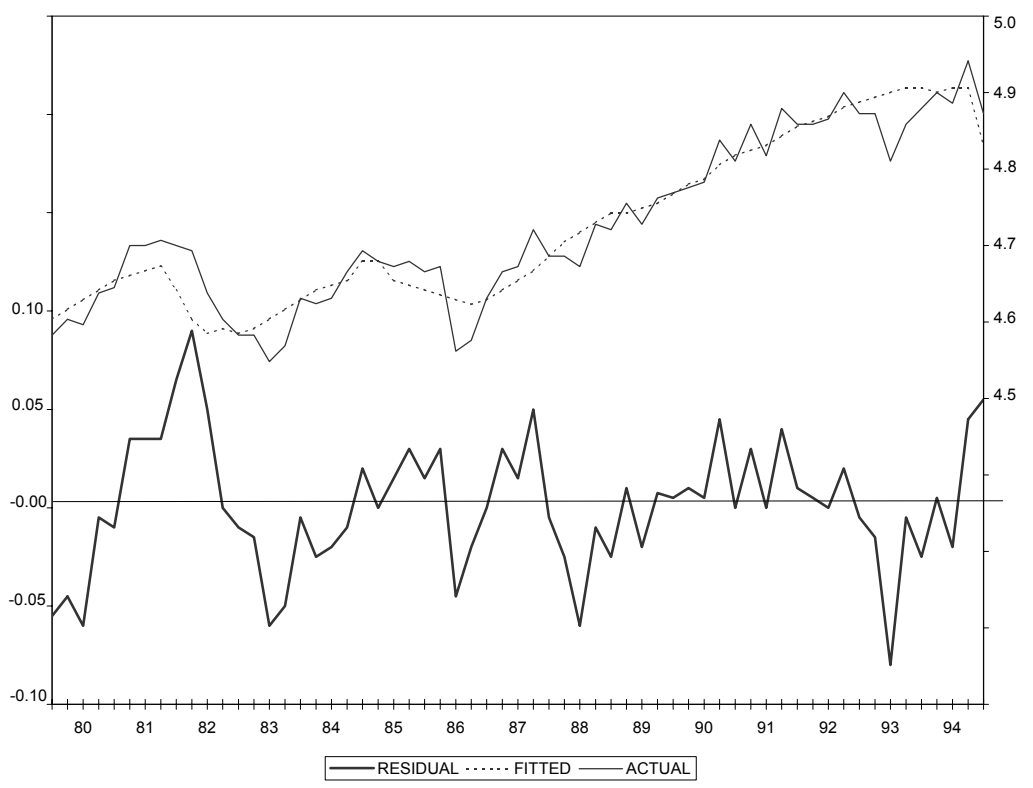


En la sección 5 se probó que el logaritmo de la producción industrial LPIND y el logaritmo del tipo de cambio real LTCRB son I(1), ahora hay que hacer 1 aprueba de que los residuales de la regresión de cointegración son $\mathrm{I}(0)$.

El cuadro 4 presenta los resultados de la prueba Dickey-Fuller aplicada a los residuales de la regresión de cointegración. La prueba se hace sucesivamente sin constante, con constante, y con tendencia lineal. En la mayoría de los casos se rechaza la hipótesis nula de que los residuales son I(1), en favor de la hipótesis alternativa de que los residuales son $\mathrm{I}(0)$. Es de hacerse notar que no se efectúa corrección por ecuaciones simultáneas, ni corrección por autocorrelación.

\section{PRONÓSTICO}

Una vez conocida la regresión de cointegración se puede utilizar para elaborar pronósticos del logaritmo de la producción industrial LPIND con base en diferentes escenarios del logaritmo del tipo de cambio real LTCRB. A continuación se presenta el escenario a partir del cual se construyen los pronósticos.

\subsection{ESCENARIO}

Para pronosticar LPIND con base en la tendencia t y en la LTCRB hay que formar un escenario con pronósticos de los componentes del tipo de cambio real bilateral.

El tipo de cambio real bilateral se define como

$$
T C R B=\frac{e P^{*}}{P}
$$

donde

$\mathrm{e}=$ tipo de cambio para valuar flujos de información bancaria (mercado controlado).

$\mathrm{P}^{*}=$ índice de precios al consumidor de Estados Unidos.

$\mathrm{P}=$ índice de precios al consumidor de México. 


\subsubsection{PRONOSTICOS DE $e$.}

Se tienen datos de $e$ hasta el primer trimestre de 1995. Para el segundo, tercero y cuarto trimestre de 1995 , se le dio al tipo de cambio el valor de 6.5 nuevos pesos.

De manera que:

\begin{tabular}{rl}
\hline & $e$ \\
\hline 95.1 & 5.967 \\
2 & 6.500 \\
3 & 6.500 \\
4 & 6.500 \\
\hline
\end{tabular}

\subsubsection{PRONÓSTICOS DE P*}

Se tienen datos $\mathrm{P}^{*}$ hasta el primer trimestre de 1995. Para pronosticar los valores de los tres trimestres restantes de 1005. se corrió una regresión de la tasa de crecimiento de $\mathrm{P}^{*}$ contra una constante y una variable dummy que toma el valor de 1 a partir del primer trimestre de 1991. Este procedimiento equivale a calcular la media de la tasa de crecimiento de $\mathrm{P}^{*}$, la cual cambia de valor a partir del primer trimestre de 1991.

La regresión es la siguiente

\section{Cuadro 5}

Regresión de Inflación de E.U.

\begin{tabular}{|c|c|c|c|}
\hline \multicolumn{4}{|c|}{ VARIABLE DEPENDIENTE $\Delta \% \mathrm{P}^{*}$} \\
\hline \multicolumn{4}{|c|}{ MUESTRA: 1987.1 A 1995.1} \\
\hline \multicolumn{4}{|c|}{ NÚMERO DE OBSERVACIONES 33} \\
\hline VARIABLE & COEFICIENTE & ERRORES EST. & ESTADÍSTICO t \\
\hline constante & 0.120 & 0.0006 & 19.0985 \\
\hline var dummy & -0.0049 & 0.0008 & -5.5742 \\
\hline R cuadrada & \multicolumn{2}{|c|}{0.5005 Media de la var dep } & 0.0095 \\
\hline R cuadrada Ajust & \multicolumn{2}{|c|}{0.4844 Desv est de la var dep } & 0.0035 \\
\hline Log Likelihood & \multicolumn{2}{|c|}{ 151.6552 Suma de resid al cuadr } & 0.0002 \\
\hline Durbin-Watson & \multicolumn{2}{|c|}{ 2.0773 Estadístico F } & 31.0722 \\
\hline
\end{tabular}


Es decir la inflación media trimestral del primer trimestre de 1987 al cuatro trimestre de 1990 fue de $1.2 \%$, y la inflación media del primer trimestre de 1991 en adelante ha sido .7\%, y este último valor es el que se toma como pronóstico.

De manera que se tiene:

\begin{tabular}{lcc}
\hline & $\Delta \% \mathrm{P}^{*}$ & $\mathrm{P} *$ \\
\hline 95.1 & .00815 & 115.467 \\
2 & .00714 & 116.291 \\
3 & .00714 & 116.291 \\
4 & .00714 & 117.958 \\
\hline
\end{tabular}

Estos pronósticos implican una inflación anual para 1995 en Estados Unidos de 2.87\%.

\subsubsection{PRONÓSTICOS DE P.}

Se tienen de $\mathrm{P}$ hasta el primer trimestre de 1995. Para pronosticar P se asignaron arbitrariamente tasa de inflación de los trimestres segundo, tercero y cuarto, de manera que la inflación anual fuera de $42 \%$.

Entonces se tiene

\begin{tabular}{rrr}
\hline & $\Delta \% \mathrm{P}^{*}$ & $\mathrm{P} *$ \\
\hline 95.1 & .097 & 112.365 \\
2 & .183 & 133.000 \\
3 & .050 & 139.650 \\
4 & .042 & 145.475 \\
\hline
\end{tabular}

\subsubsection{PRONÓSTICOS DE TCRB}

Los pronósticos del tipo de cambio real bilateral se calculan a partir de los pronósticos anteriores, como se muestra a continuación 


\begin{tabular}{rrrrr}
\hline \multicolumn{2}{r}{$\mathrm{e}$} & \multicolumn{2}{c}{$\mathrm{P} \mathrm{P}^{*}$} & \multicolumn{2}{c}{$\mathrm{TCRB}$} \\
\hline 95.1 & 5.967 & 115.467 & 112.365 & 6.1317 \\
2 & 6.500 & 116.291 & 133.000 & 5.6834 \\
3 & 6.500 & 117.122 & 139.650 & 5.4514 \\
4 & 6.500 & 117.958 & 145.475 & 5.2705
\end{tabular}

\subsection{PRONÓSTICOS DE LPIND}

Una vez que se tienen los pronósticos del logaritmo del tipo de cambio real LTCRB, se puede utilizar la regresión de cointegración para pronosticar el logaritmo de la producción industrial LPIND. Los pronósticos son los siguiente.

\begin{tabular}{rrc}
\hline & LPIND & PIND \\
\hline 95.1 & 4.8761 & 131.1220 \\
2 & 4.8435 & 126.9191 \\
3 & 4.8569 & 128.6354 \\
4 & 4.8688 & 130.1713 \\
\hline
\end{tabular}

La gráfica 11 presenta los valores observados de LTCRB y los valores pronosticados.

\section{Gráfica 11}

Valores Observados y Pronósticos del LTCRB

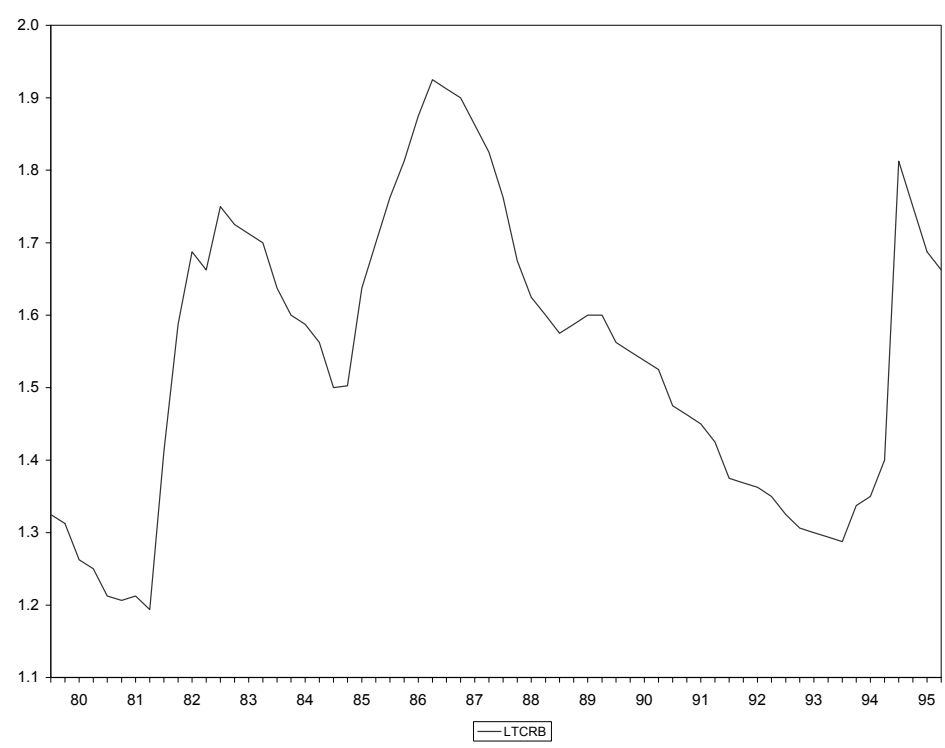


Los pronósticos trimestrales implican una variación porcentual anual del índice de volumen de la producción industrial de $-4.19 \%$.

\subsection{PRONÓSTICOS DE LPIB}

Como se vio en la sección 3 existe una correlación alta entre el logaritmo de la producción industrial LPIND y el logaritmo del producto interno bruto. Mas específicamente se corrió la siguiente regresión del logaritmo del PIB trimestral contra dos variables dummy estacionales y LPIND.

Con la regresión anterior y con los pronósticos de LPIND, se calcularon pronósticos del logaritmo del PIB para los cuatro trimestres de 1995. Los resultados son los siguientes.

\begin{tabular}{rcc}
\hline & LPIB & PIB \\
\hline 95.1 & 8.6228 & 5557.130 \\
2 & 8.6259 & 5574.534 \\
3 & 8.6086 & 5479.094 \\
4 & 8.6534 & 5729.692 \\
\hline
\end{tabular}

Los pronósticos trimestrales implican una variación porcentual anual del PIB de $-4.65 \%$ en 1995 .

\section{Cuadro 6}

\section{Regresión del PIB}

\begin{tabular}{|c|c|c|c|}
\hline \multicolumn{4}{|c|}{ VARIABLE DEPENDIENTE LPIB } \\
\hline \multicolumn{4}{|c|}{ MUESTRA 1980.1 A 1994.4} \\
\hline \multicolumn{4}{|c|}{ NÚMERO DE OBSERVACIONES 60} \\
\hline VARIABLE & COEFICIENTE & ERROR ESTAND & ESTADISTICO $\mathrm{t}$ \\
\hline Constante & 5.0210 & 0.1100 & 45.6519 \\
\hline Var dummy2 & 0.0272 & 0.0060 & 4.5506 \\
\hline Var dummy 4 & 0.0360 & 0.0060 & 5.9976 \\
\hline LPIND & 0.7387 & 0.0233 & 31.6604 \\
\hline R cuadrada & \multicolumn{2}{|c|}{0.9518 Media de la var dep } & 8.5256 \\
\hline $\mathrm{R}$ cuadrada ajust & \multicolumn{2}{|c|}{0.9492 Desv est de la var dep } & 0.0837 \\
\hline Log Likelihood & \multicolumn{2}{|c|}{ 155.214 Suma de resid al cuad } & 0.0199 \\
\hline Durbin-Watson & \multicolumn{2}{|c|}{0.7158 Estadístico F } & 368.7512 \\
\hline
\end{tabular}




\section{CONCLUSIONES}

A partir de un modelo sencillo de equilibrio general se derivó una relación inversa entre el producto y el tipo de cambio real.

Tanto el índice de volumen de la producción industrial como el tipo de cambio real bilateral MéxicoEstados Unidos resultan ser variables no-estacionarias. Más aún, ambas variables están cointegradas.

La regresión de cointegración se utilizó para formar pronósticos trimestrales del índice de volumen de la producción industrial y del producto interno bruto para 1995. Las variaciones porcentuales pronosticadas para 1995 son $-4.19 \%$ para el IVPI, y de $-4.65 \%$ para el PIB. 


\section{BIBLIOGRAFÍA}

Engle, R.F. y Granger, C.W.J., Long-run Economic Relationships: Readings in Cointegration, Oxford: Oxford University Press, 1991.

Mussa, M., "The Effects of Commercial, Fiscal, and Exchange Rate Policies on the Real Exchange Rate" en S. Edwards y L. Ahamed, Economic Adjustment and Exchange Rates in Developing Countries, Chicago: The University of Chicago Press, 1986. 